\title{
Extra-Nodal Non-Cutaneous Peripheral T cell Lymphoma: Common Peripheral Blood Findings and Clinical Follow Up
}

\author{
Hongwei Ma, Catherine Xie, Mohamed Masoud, Robin Williams and Ming Xie*
}

Department of Pathology, OUWB School of Medicine, William Beaumont Hospital, USA

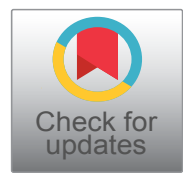

*Corresponding author: Dr. Ming Xie, Department of Pathology, OUWB School of Medicine, William Beaumont Hospital, 44201 Dequindre Road, Troy, MI 48084-1198, USA, Tel: 248-964-4109, Fax: 248-964-4110

\begin{abstract}
Peripheral $\mathrm{T}$ cell lymphoma $(\mathrm{TCL})$ is relatively uncommon and may involve lymph node, skin and extra-nodal non-cutaneous organs. Nodal TCL often presents as systemic disease with unfavorable outcomes. Extra-nodal non-cutaneous TCL, however, may remain as localized disease and be treated differently with favorable prognosis. This study revealed the common peripheral blood findings and clinical follow ups in patients with extra-nodal non-cutaneous mature TCL. There were 9 patients, 5 males and 4 females, median age 52, including 1 extra-nodal NK/T cell lymphoma, nasal type; 1 anaplastic large cell lymphoma, ALK-negative; 1 enteropathy associated T cell lymphoma and 6 peripheral TCL, not otherwise specified. No patients showed concurrent bone marrow, lymph node or cutaneous involvement by combined pathological and clinical studies. Seven patients had anemia; with associated lymphopenia in 2 patients, thrombocytopenia and thrombocytosis in 1 patient each. Three patients received CHOP chemotherapy; 1 with radiation therapy and subsequent chemotherapy; 4 with surgical procedures and 1 with conservative management only. Two patients died of disease after chemotherapy. Seven patients were in remission or alive with disease. Anemia was common in patients with extra-nodal non-cutaneous TCL, which made the patient management more challenging. In comparison with nodal TCL, extra-nodal non-cutaneous TCL in this study remained as localized disease without systemic involvement. Clinical management was more variable with fewer patients receiving chemotherapy. Surgical treatment alone and conservative management were the treatment of choice in more patients with relatively favorable clinical outcome.
\end{abstract}

\section{Keywords}

Peripheral T cell lymphoma, Anemia, Complete blood count, Bone marrow biopsy

\section{Introduction}

Peripheral T cell lymphoma (TCL) is an uncommon disease and accounts for approximately $6 \%$ of all lymphomas in US $[1,2]$. Unlike many B-cell lymphomas, which have shown recent declined incidence in US, the incidence for T-cell lymphomas has continued to rise $[3,4]$. Based on the organ system involved, T-cell lymphomas can be further divided into nodal, extranodal, and cutaneous subtypes with different incidence rate $[5,6]$. In recent years, there have been significant advances in our understanding of TCL in its molecular mechanism, lymphoma classification, treatment strategy and long term prognoses $[7,8]$.

In comparison with TCL involving lymph node and skin, extra-nodal non-cutaneous $T$ cell lymphoma may have significantly different clinical presentation and require different therapeutic approaches. Due to its rarity, however, extra-nodal non-cutaneous TCL is still not well studied, which brings challenges to medical care providers both in the diagnosis and management of this disease.

A complete blood count $(\mathrm{CBC})$ is one of the most common laboratory tests in medical practice [9]. Abnormal peripheral blood findings have been shown to be an unfavorable prognostic factor in patients with lymphoma [10-12]. This study revealed the common peripheral blood findings in patients with extra-nodal non-cutaneous $T$ cell lymphoma as a group of disease with clinical correlation and follow up evaluations.

Citation: Ma H, Xie C, Masoud M, Williams R, Xie M (2019) Extra-Nodal Non-Cutaneous Peripheral T cell Lymphoma: Common Peripheral Blood Findings and Clinical Follow Up. Int J Pathol Clin Res 5:098. doi.org/10.23937/2469-5807/1510098

Accepted: September 05, 2019: Published: September 07, 2019

Copyright: (c) $2019 \mathrm{Ma} \mathrm{H}$, et al. This is an open-access article distributed under the terms of the Creative Commons Attribution License, which permits unrestricted use, distribution, and reproduction in any medium, provided the original author and source are credited. 


\section{Materials and Methods}

\section{Patient selection and general information}

Pathological reports filed at the Department of $\mathrm{Pa}$ thology, William Beaumont Hospital - Troy from 2006 to 2016 were reviewed. In total, 9 patients with extra-nodal non-cutaneous TCL were identified. General patient information including age and gender was collected, together with the related pathological diagnosis and laboratory test results.

\section{Routine histologic examination of biopsy and ex- cisional specimens and peripheral blood evalua- tions}

Core biopsy and excisional specimens were processed following the standard procedures. Hematoxylin and Eosin (H\&E) stained slides were prepared for microscopic examination. Giemsa stained smears from the bone marrow aspirate and Wright stained smears from peripheral blood samples were prepared for cytological evaluation in patients with bone marrow biopsies for lymphoma staging evaluation. The diagnosis of $\mathrm{T}$ cell lymphoma was made according to the recent WHO classification system [13].

CBC data at or near the time of TCL diagnosis was available for review in all patients. Peripheral blood smears were also prepared for concurrent morphological examination with $\mathrm{CBC}$ results.

\section{Flow cytometry and immunohistochemistry}

Fresh tumor tissue was available in 1 patient for flow cytometry study. Multicolor flow cytometry evaluation was focused on identifying evidence of lymphoma, leukemia and myelodysplasia. A more sensitive and specific $T$ cell receptor- $V$ beta (TCR- $V$ beta) panel was added since 2010. Immunohistochemical studies were performed following the standard protocols on all histological specimens with adequate controls.

$T$ cell receptor (TCR) gene rearrangement and Epstein Barr virus (EBV) RNA detection by in-situ hybridization

TCR gene rearrangement studies were performed by polymerase chain reaction (PCR) on paraffin imbedded formalin fixed tumor tissue to confirm the diagnosis of T cell lymphoma in some patients. Three in-house validated multiplexed primer sets spanning the variable and joining regions of the T-cell receptor gamma (TCR-gamma) chain gene were used. EBV infection was determined by Epstein-Barr encoding region (EBER) in situ hybridization on paraffin sections by using the Leica Microsystems (Leica Microsystems, Bannockburn, IL 60015) fluorescein labeled EBER probe (ISH5687-A) and the Bond Polymer refine detection kit (DS9800) detecting latent EBV infection.

\section{Clinical management and treatment received with follow ups}

Retrospective review of the electronic medical charts was performed. Clinical management data was collected, including clinical presentation and assessment, physical and imaging studies, previous medical and surgical history, treatment received and outcomes with clinical follow ups for at least 12 months after the diagnosis of TCL was made.

\section{Results}

\section{General patient information and clinical presen- tation}

There were 9 patients, including 5 males and 4 females, with a median age of 52 (33-96). All patients were found to have a mass lesion by imaging and clinical evaluations. Four patients had excisional specimens and the other 5 patients had core biopsies for pathological examination. Thorough clinical and imaging evaluations revealed no evidence of concurrent lymph node, bone marrow or cutaneous involvement of T cell lymphoma. Bone marrow biopsy was performed in 4 patients for lymphoma staging evaluation.

\section{Pathological examination, immunophenotyping studies and diagnosis}

Histological examination of the biopsy and excisional specimens revealed atypical lymphoid infiltrate with disruption of the normal tissue structure, indicative of lymphoma. Flow cytometry and immunohistochemical studies identified abnormal clonal $\mathrm{T}$ cell populations in all specimens. The histological and immunophenotypic findings were consistent with TCL. Five patients had specimens sent for TCR gene rearrangement studies and were positive for monoclonal TCR-gamma, which further confirmed the diagnosis. EBER was interpreted as positive by in situ hybridization in 2 of the 5 patients studied.

The diagnosis of TCL was shown in summary Table 1. Six patients were diagnosed of peripheral TCL, not otherwise specified; 1 extra-nodal NK/T cell lymphoma, nasal type; 1 anaplastic large cell lymphoma, ALK-negative and 1 enteropathy associated T cell lymphoma.

\section{Bone marrow biopsy results}

Bone marrow biopsy specimens included core biopsies, aspirate smears and peripheral blood smears. In addition to routine histological and cytological evaluations, all bone marrow biopsy specimens were sent for flow cytometry, immunohistochemistry and cytogenetic studies, and revealed no evidence of TCL. No other hematopoietic or non-hematopoietic disorders were diagnosed.

\section{Abnormal peripheral blood findings}

All patients had peripheral blood samples sent for 

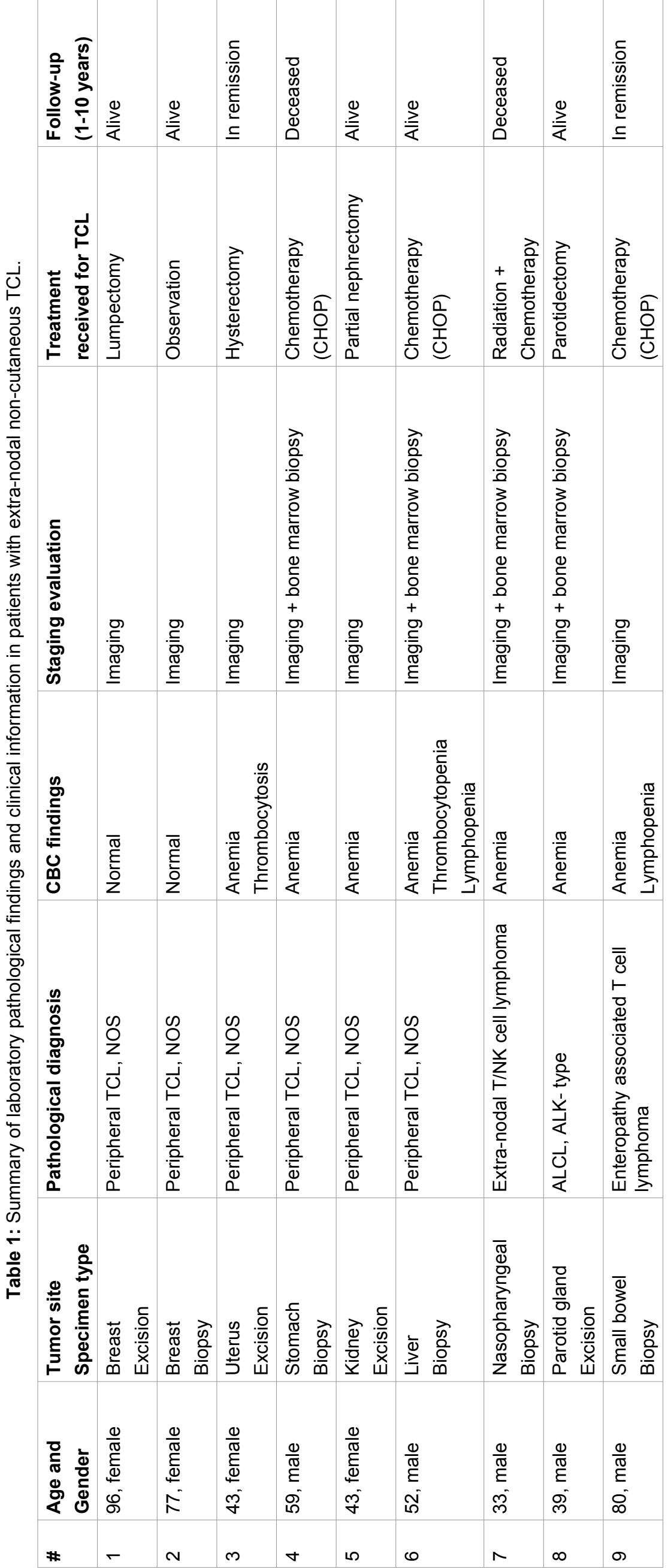
automated CBC analyses. Peripheral blood smears were also available for morphological confirmation. Seven patients had abnormal CBC findings and 2 showed normal concurrent $\mathrm{CBC}$ parameters. The anemia in 7 patients was normocytic and normochromic. Two patients also had lymphopenia, 1 had thrombocytopenia and another had thrombocytosis. No lymphocytosis was seen in any patient and no abnormal circulating lymphocytes were identified on peripheral blood smear review.

\section{Clinical management and follow up}

As shown in Summary Table 1, 3 patients received standard chemotherapy (CHOP) and 4 had surgical excisions. One patient treated with radiation therapy followed with chemotherapy and 1 was followed with observation only. Clinical management in these patients also included treatment for the comorbidities including anemia.

Two of the patients (\#4 and \#7) received chemotherapy died of disease and related complications. The other 7 patients were in remission or alive with disease during the follow up period. Patient \#4 experienced multiple chemotherapy related complications, including pancytopenia and septic shock, and subsequently died of multi-organ failure.

Patient \#7 received radiation therapy followed with chemotherapy. Despite treatments, the lymphoma showed local progression with mediastinum involvement pressing on right main airway. Patient died of complications related to pneumonia, acute respiratory failure and tracheostomy site bleeding.

\section{Discussions}

Unlike its counterpart in lymph node, extra-nodal non-cutaneous TCL was more likely to be localized disease without systemic involvement. However, most patients (7/9) in this study still had abnormal CBC findings. Anemia was the most common, followed by lymphopenia, thrombocytopenia and thrombocytosis. Anemia has been reported to carry a negative impact on patient survival and bring more challenge and complexity in patient management $[14,15]$. However, the clinical significance of anemia in this patient group was unclear and the pathogenesis of abnormal CBC in patients with extra-nodal non-cutaneous TCL still needs further investigation.

Due to its rare incidence, extra-nodal non-cutaneous $\mathrm{TCL}$ usually comes as an unanticipated diagnosis. Even presenting as a mass lesion, the clinical suspicion for lymphoma was low and rarely had fresh tissue for lymphoma evaluation by flow cytometry. In this study only 1 of the 9 patients had fresh tissue obtained for flow cytometry at the time of surgery. Immunohistochemical studies were essential to establish the diagnosis of TCL. TCR gene rearrangement study was often needed for the diagnosis confirmation in challenging cases.
The standard treatment for TCL is still lacking $[16,17]$. Treatment in the front-line setting for nodal TCL often consists of cyclophosphamide, doxorubicin, vincristine, and prednisone (CHOP) or CHOP-like regimens [18,19]. The clinical management of extra-nodal non-cutaneous $\mathrm{TCL}$ was more variable with fewer patients receiving chemotherapy. Based on its anatomic location and localized diseases, surgical treatment alone and conservative management were the treatment of choice in more patients with extra-nodal non-cutaneous TCL.

The long term survival for patients with nodal TCL is often poor. Patients may experience treatment failure and frequent disease relapse $[20,21]$. Primary cutaneous TCL has distinct clinical outcomes in comparison with systemic TCL with cutaneous involvement. For TCL limited to skin, topical therapies or skin-directed therapies were favored over multiagent chemotherapy (CHOP) and showed similar disease-free or overall survival with significantly less toxicity [22-24]. In this study, 4 patients received CHOP/CHOP-like chemotherapy, and 2 of them died of disease and another 2 in remission. There was no mortality in patients only with surgery and conservative management. Chemotherapy did not show more favorable outcome in the overall disease survival in this study. The anatomic location and localized disease seem to be the more important factors for the long term prognosis in these patients.

\section{Conclusions}

Extra-nodal non-cutaneous $\mathrm{TCL}$ is a rare disease. Abnormal $\mathrm{CBC}$ findings were common and might bring more challenges in patient management, although its impact on patient long term survival was unclear. Surgical excision and conservative management were the treatment of choice in more patients with favorable prognosis. Traditional chemotherapy (CHOP) did not improve the overall survival rate. The favorable clinical outcome was likely due to its anatomic location and localized disease. However, the authors acknowledge that the number of patients in this study is limited and more studies are needed for this rare disease.

\section{Acknowledgement}

Department of Pathology, OUWB School of Medicine, William Beaumont Hospital.

\section{References}

1. Wang SS, Vose JM (2013) Epidemiology and prognosis of T-Cell lymphoma. In: Foss F, T-Cell Lymphomas, Contemporary Hematology. Humana Press, Totowa, NJ, USA, 25-39.

2. Masoud M, Xie C, Zhou J, Chen X, Xie M (2018) Clinical pathological features of peripheral $T$ cell lymphoma with concurrent bone marrow involvement. Am J Clin Exp Med 6: 22-26.

3. Criscione VD, Weinstock MA (2007) Incidence of Cutaneous T-Cell Lymphoma in the United States, 1973-2002. Arch Dermatol 143: 854-859. 
4. Zucca E (2008) Extranodal lymphoma: A reappraisal. Annals of Oncology 19: iv77-iv80.

5. Foss FM, Zinzani PL, Vose JM, Gascoyne RD, Rosen ST, et al. (2011) Peripheral T-cell lymphoma. Blood 117: 67566767.

6. Vose J, Armitage J, Weisenburger D, International T-Cell Lymphoma Project (2008) International peripheral T-cell and natural killer/T-cell lymphoma study: Pathology findings and clinical outcomes. J Clin Oncol 26: 4124-4130.

7. Broccoli A, Zinzani PL (2017) Peripheral T-cell lymphoma, Not otherwise specified. Blood 129: 1103-1112.

8. de Leval L, Bisig B, Thielen C, Boniver J, Gaulard P (2009) Molecular classification of T-cell lymphomas. Crit Rev Oncol Hematol 72: 125-143.

9. Tefferi A, Hanson CA, Inwards D (2005) How to interpret and pursue an abnormal complete blood cell count in adults. Mayo Clin Proc 80: 923-936.

10. Conlan MG, Armitage JO, Bast M, Weisenburger DD (1991) Clinical significance of hematologic parameters in nonHodgkin's lymphoma at diagnosis. Cancer 67: 1389-1395.

11. Hohaus S, Massini G, Giachelia M, Vannata B, Bozzoli V, et al. (2010) Anemia in Hodgkin's lymphoma: The role of interleukin-6 and hepcidin. J Clin Oncol 28: 2538-2543.

12. Linaburg T, Davis AR, Frey NV, Landsburg DJ, Schuster SJ, et al. (2018) Classical Hodgkin lymphoma patients have an increased incidence of idiopathic acquired aplastic anemia. Blood 132: 5098

13. Swerdlow SH, Campo E, Harris NL, Jaffe ES, Pileri SA, et al. (2017) WHO Classification of Tumours of Haematopoietic and Lymphoid Tissues. IARC, Lyon, France.

14. Stauder R, Peter Valent P, Theurl I (2018) Anemia at older age: Etiologies, clinical implications, and management. Blood 131: 505-514.

15. Birgegård G (2008) Managing anemia in lymphoma and multiple myeloma. Ther Clin Risk Manag 4: 527-539.

16. Carson KR, Horwitz SM, Pinter-Brown LC, Rosen ST, Pro B, et al. (2017) A prospective cohort study of patients with peripheral T-cell lymphoma in the United States. Cancer 123: 1174-1183.

17. Laribi K, Alani M, Truong C, de Materre AB (2018) Recent advances in the treatment of peripheral T-cell lymphoma. Oncologist 23: 1039-1053.

18. Briski R, Feldman AL, Bailey NG, Lim MS, Ristow K, et al. (2014) The role of front-line anthracycline-containing chemotherapy regimens in peripheral T-cell lymphomas. Blood Cancer J 4: e214.

19. Mercadal S, Briones J, Xicoy B, Pedro C, Escoda L, et al. (2008) Intensive chemotherapy (high-dose CHOP/ESHAP regimen) followed by autologous stem-cell transplantation in previously untreated patients with peripheral T-cell lymphoma. Ann Oncol 19: 958-963.

20. Weisenburger DD, Savage KJ, Harris NL, Gascoyne RD, Jaffe ES, et al. (2011) Peripheral T-cell lymphoma, not otherwise specified: A report of 340 cases from the international peripheral T-cell lymphoma project. Blood 117: $3402-3408$

21. Wilhelm M, Smetak M, Reimer P, Geissinger E, Ruediger T, et al. (2016) First-line therapy of peripheral T-cell lymphoma: Extension and long-term follow-up of a study investigating the role of autologous stem cell transplantation. Blood Cancer J 6: e452.

22. Wilcox RA (2016) Cutaneous T-cell lymphoma: 2016 update on diagnosis, risk-stratification, and management. Am J Hematol 91: 151-165.

23. Korgavkar K, Xiong M, Weinstock M (2013) Changing incidence trends of cutaneous T-cell lymphoma. JAMA Dermatol 149: 1295-1299.

24. Bagherani N, Smoller BR (2016) An overview of cutaneous T cell lymphomas. F1000Res. 